\title{
SYMPOSIUM
}

\section{The value of mass screening for chlamydia control in high prevalence communities}

\author{
S Hodgins, R W Peeling, S Dery, F Bernier, A LaBrecque, J-F Proulx, J Joly, M Alary, \\ D Mabey
}

Sex Transm Infect 2002;78(Suppl I):i64-i68

The social context and epidemiology of STIs in remote communities in Northern Canada was examined. These communities have a persistently high reported incidence of gonorrhoea and chlamydial infection. They remain in the hyperendemic phase of the $\mathrm{N}$ gonorrhoeae and $C$ trachomatis epidemics. They are ethnically and culturally homogeneous and contain no readily identifiable core groups, making it impossible to distinguish between spread and maintenance networks. Mass screening of the adult population can reduce the reservoir of $C$ trachomatis infection under these circumstances. It is particularly important to target men in settings such as this where women are routinely screened in antenatal and family planning clinics.

See end of article for authors' affiliations

.......................

Correspondence to: Dr Rosanna W Peeling, Sexually Transmitted Diseases Diagnostics Initiative, UNDP/World Bank/WHO Special Programme on Research and Training in Tropical Diseases, World Health Organisation, 20 Avenua Appia, 1211 Geneva 27

Switzerland; peelingr@who.int

Accepted 30 November 2001

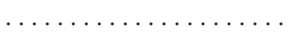

- pidemics of sexually transmitted infection (STI) evolve through predictable phases, - shaped by a dynamic interplay between the pathogen, the behaviours of the subpopulations in which it emerges, and the prevention efforts that are developed to limit its impact. ${ }^{1}$ In planning appropriate control strategies for particular STIs in particular populations, it is important to consider the phase which the epidemic has reached.

In this paper we describe the social context and epidemiology of STIs in remote Inuit communities in Northern Quebec, and the impact of a mass screening campaign for Chlamydia trachomatis, assessed by means of a community randomised trial. Reported rates of chlamydial infection and gonorrhoea in these remote communities are 20 to 50 times higher than in Quebec Province as a whole, in spite of the availability of the same high quality free medical services. Following an intensive health education campaign and condom promotion, the incidence of gonorrhoea has declined somewhat in the past decade, but the reported incidence of chlamydial infection has remained consistently high, in spite of a screening programme targeting women attending for antenatal care, family planning, or cervical cytology. In terms of the dynamic topology model of STI transmission, ${ }^{1}$ these communities were in the hyperendemic phase of the $C$ trachomatis epidemic (phase II) when this study began, but in the early intervention or decline phase of the $N$ gonorrhoeae epidemic (phase II' or III).

\section{SOCIAL CONTEXT \\ Geography}

The Nunavik region of northern Quebec is made up of 14 coastal communities situated on the shores of Hudson's Bay and Ungava Bay, covering a total area of $560000 \mathrm{~km}^{2}$. The population is almost exclusively Inuit except in the communities of Kuujjuaq and Kuujjuaraapik. Village size varies from 120 to 1800 in total population. Villages are only accessible by air, except for several sea shipments arriving during summer months.

\section{History}

The way of life of these communities has changed enormously in the past century. ${ }^{2}$ The fur trade became established in the late 19th century, providing access to a cash economy. This began to displace the traditional subsistence economy which depended on hunting and fishing. However, the economy remained heavily dependent on the caribou, and a drastic decline in herd numbers in the early 20th century led to widespread hardship and food shortages. The fur trade collapsed with the depression in the 1930s, depriving the Inuit of their only source of cash. The resulting poverty and epidemics of tuberculosis and other infectious diseases led to a very high infant and childhood mortality in the 1930s and 1940s, which remains evident in the existing population structure.

Medical services were introduced by the government in the late 1940s in response to the TB epidemic. A mass screening programme led to a diagnosis of TB in almost $20 \%$ of the population, and infected individuals were evacuated to the south for treatment, which often lasted several years. The family allowance programme was extended to the region at about this time, and the federal government began to encourage the settlement of the Inuit population in villages. An extensive construction programme was started in the 1960s, and schools and nursing stations were established in most of the communities. Families were compelled to send their children to school, which meant that the time available to spend in hunting camps was limited, and that the traditional way of life was increasingly replaced by a routine and village environment largely imposed by outsiders. A 1992 survey found that 25\% of women under 45 years had never participated in the traditional way of life.

\section{Demographics}

In 1991 the total population of the region was $7660,90 \%$ of whom were Inuit. ${ }^{2}$ The population 


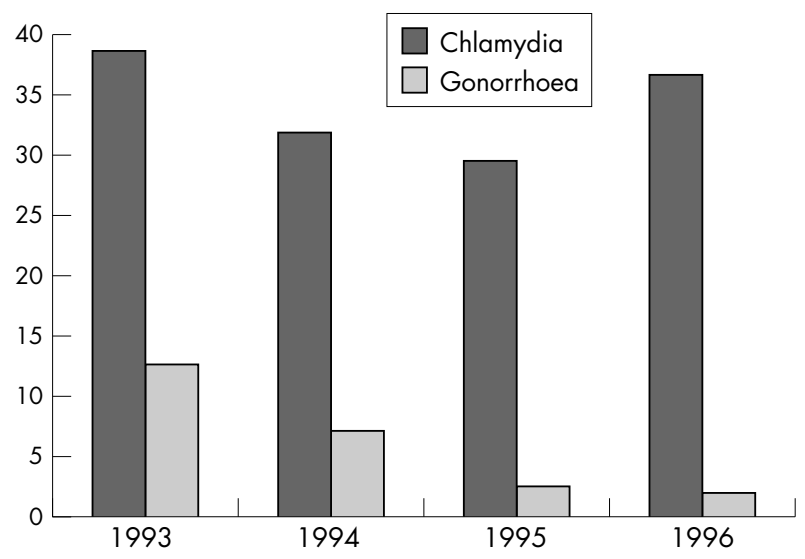

Figure 1 Reported chlamydia and gonorrhoea rates per 1000 population per year in Nunavik, 1993-6.

has almost quadrupled since 1950, and continues to grow at $3 \%$ a year, although the annual general fertility rate has fallen from approximately 200 per 1000 women aged 15-49 in the 1960 s to 140 per 1000 since the 1970s. Twenty five per cent of births are to teenage mothers, and $14 \%$ to girls aged less than 18. Forty per cent of the population are aged less than 15 years, and only $2 \%$ more than 65 years. The median age of the population is 18 years (compared with 34 years for Quebec province as a whole) (fig 1 ).

Rapid population growth has led to a shortage of housing and overcrowded living conditions. The average household contains five individuals and $40 \%$ of households have six or more persons. It has also led to high levels of unemployment, particularly among the young: the rate in the 15-24 age group is 20 times higher in these communities than in the province of Quebec as a whole.

\section{Social/political/economic situation}

In Inuit society the extended family and camp group (or clan) has been the fundamental social unit until very recently. It remains important, but social bonds have been weakened in the past 40 years with the enforced movement from the camp to the larger community of a settled village, and by outside influences which have promoted individuality at the expense of communal values. People were housed in villages mainly as nuclear families, although often with a grandparent or other relative in the house as well. It remains common for members of the extended family, even when living in different houses, to gather in one household daily to share the main midday meal.

Interaction between individuals of the same age group has become more common. For example, it is now usual for groups of young men to go hunting together, whereas in the past this would have been a family or clan activity. Hockey has become very popular and brings young people together in an activity with a strong social dimension. Fewer people are marrying, and the majority of cohabiting young adults are unmarried. The majority of first children are born to single teenage mothers, and some $30 \%$ of children are given up for adoption, although adoptive parents are often relatives of the mother.

Rapid social changes and the breakdown of the traditional way of life have imposed severe stresses on these communities, and this is reflected in high rates of substance abuse, violence, and suicide. By the late 1960s, alcohol use had become widespread, and drugs (mainly cannabis) became more readily available. Use of these substances was taken up particularly by young people, and the pattern of use was potentially destructive. Twenty five per cent of adults are regular at-risk drinkers-that is, they drink at least once a month and have five or more drinks per session-and a similar percentage report having had alcohol related problems in the past year (for example, injuries, fights, or problems with the police, at work, or at school). Thirty eight per cent of adults report having smoked cannabis in the previous year ( $47 \%$ of men, $30 \%$ of women). Almost 10\% of individuals of both sexes aged 15-24 sniff solvents, and more than $10 \%$ of the male population aged 15-24 have taken cocaine. ${ }^{2}$

Domestic and sexual violence is common. Eighty three per cent of adults over 45 years interviewed in 1992 felt that physical or verbal abuse of wives (or husbands) was a serious problem in their communities. More than 50\% of women under 25 reported having been sexually assaulted or abused -that is, they replied "yes" to the question: "Have you ever been forced into any kind of sexual activity (kissing, fondling, touching, or intercourse)?". ${ }^{2}$ In one of these communities, 127 cases of childhood sexual abuse were reported to the Youth Protection Authority in 1993.

The 1980s ended with a marked increase in the rate of suicide in young people-the rate rose to 80/100 000 total population, and in the 15 to 24 age group it was 20 times higher than the overall rate in Quebec Province. A survey conducted in the early 1990s found that more than a quarter of young people aged 15 to 24 years had attempted suicide. ${ }^{2}$ Risk factors included a history of solvent abuse, experience of having been physically abused, and having a parent with a substance abuse problem.

\section{Sexual networks, behaviour and attitudes}

There is no known commercial sex in these communities, which are ethnically and culturally homogeneous and lack clearly identifiable core groups. In small communities such as these it is not possible to distinguish spread from maintenance networks.'

A survey conducted in 1992 found that 22\% of men and 17\% of women reported more than one sexual partner in the past year, as did $32 \%$ of those aged 15 to $24 .^{2}$ Eighty per cent of adults had not used any form of contraception in the past year, although it was more commonly used by young people, in whom the condom was the most popular method. Among those who reported two to four partners in the past year, 37\% had used condoms at least some of the time, but only $23 \%$ of those with five or more partners had used condoms. The number of condoms distributed through the nursing stations has increased greatly since 1992.

In a study of women's perception of STI in the region, they reported feeling scared, ashamed, confused, and depressed when told that they have an STI; but they often felt powerless to do anything about it. ${ }^{3}$ Some of them attributed the high rates of STI to "contamination" from the outside world, which had disrupted the equilibrium between health and disease in traditional societies. The women knew "careless behaviour," or having many sexual partners were often associated with increased risk of acquisition of STIs. Every woman interviewed was knowledgeable about the use of condoms to prevent transmission of STIs, but some expressed concern and anger that their male partners were unwilling to use them. They had all heard of "the incurable STI" and were concerned that "AIDS would spread like wildfire here if somebody had it, because there's a lot of people who are just having sex with many different partners and they're not careful about condoms, they're not using condoms."

\section{Basic public health organisation and infrastructure}

The community is served by two small hospitals which are located in the two major transportation centres. The two hospitals each have a laboratory capable of performing serology for syphilis, microscopy and culture for gonorrhoea, microscopy for Trichomonas vaginalis, bacterial vaginosis, and candidiasis, and enzyme immunoassay (EIA) for $C$ trachomatis. Each community is served by a nursing station or health centre, 
staffed by a public health nurse or nurse practitioner with support from the physicians and nursing staff of the two hospitals. As elsewhere in Canada, there is universal access to health care, and STI diagnosis and treatment are provided free of charge.

\section{THE STI PREVENTION PROGRAMME}

Community leaders in Nunavik have been sensitised to STI and HIV risk for several years. A well attended regional conference was held in 1993. Since then, an active campaign of condom distribution has been under way and the volume of condoms distributed in the region has increased severalfold. At the clinical level, screening of young women has been widespread since the early 1990s-all women attending for PAP smears or antenatal care have been offered screening for gonorrhoea (by culture of cervical swab) and chlamydial infection (by EIA of cervical swab). In recent years, contact tracing has been more systematic.

\section{Surveillance activities}

The most common STIs seen in Nunavik are gonorrhoea, chlamydial infection, trichomoniasis, and genital warts. Only the first two are reportable, so no data are available for trichomoniasis or warts. Gonorrhoea and chlamydial infections are reported by the laboratory. In addition to women attending for PAP smear and antenatal care, men and women presenting with symptoms suggestive of STIs are also tested. An unlinked anonymous HIV screening programme conducted in pregnant women between 1989 and 1993, which achieved a coverage of $90 \%$, found no HIV positive cases among almost 1000 tested. $^{2}$

\section{STI epidemiology}

The reported rates for chlamydia and gonorrhoea for the region were 3500 and 1000 per 100000 population in 1990, approximately 20 and 50 times higher, respectively, than the rates for the rest of the province. The highest chlamydia rates were in the 20 to 24 year olds ( 12500 per 100000 ) and the 15 to 19 year olds ( 10250 per 100000 ). The highest gonorrhoea rates were in 20 to 24 year old women ( 4500 per 100000 ) and men (4050 per 100000 ). Perhaps as a result of the health education programme, gonorrhoea rates fell substantially during the early 1990s, but chlamydial rates have remained high. This difference may be explained by the greater proportion of asymptomatic chlamydial infections, or by relatively poor compliance with the seven day treatment regimen recommended for chlamydial infection at that time. Six monthly reported rates of gonorrhoea and chlamydial infection from 1993 to 1996 are shown in fig 1 .

Ectopic pregnancy is an important complication of gonorrhoea and chlamydial infection in women. The ectopic rate is almost four times as high in Nunavik as in the province of Quebec as a whole (314 cases per 100000 total population per year, compared with 87 for Quebec). As surgical facilities are not available locally, patients have to be flown to Montreal, which increases the risk of complications and entails considerable expense ( $\$ 25000$ for each airlift alone). There is thus a strong economic argument for improved control of these infections. Greater resources for STI control can also be justified as a strategy for HIV prevention, as there is compelling evidence that other STIs facilitate the heterosexual transmission of HIV. ${ }^{4}$

The $C$ trachomatis epidemic has remained in the hyperendemic phase in these communities in spite of the health promotion and screening programmes introduced in the early 1990s. In 1994, in an attempt to bring it under control, a community based screening programme was promoted in one village (population 420) on the Ungava Bay coast. All adults were encouraged to come to the clinic to be tested, and particular efforts were made to encourage men to do so. Ninety per cent of adults were screened (by EIA on a urethral or cervical swab), and the prevalence of chlamydial infection was found to be approximately 10\%. In 1994 and 1995 the reported rate fell dramatically, but it increased again in 1996.

Since 1994, nucleic acid amplification tests have become widely available for the diagnosis of $C$ trachomatis infection. These are more sensitive than the older antigen detection assays, and have the added advantage of using non-invasive samples, such as first catch urine. This provided an opportunity to extend an improved community based mass screening programme to other communities in Nunavik, and to determine its impact.

\section{RANDOMISED STUDY OF MASS SCREENING FOR C TRACHOMATIS}

In the spring of 1996, all 14 communities were invited to participate in a mass chlamydia screening campaign, using polymerase chain reaction (PCR) to detect the infection in first catch urine samples. The aims of the project were explained to the municipal council and health committees. When told of the success of the previous screening programme, they all expressed a desire to be included. Communities were paired, trying to match for population size and reported chlamydia rate, and to allow a roughly even split of intervention and comparison communities between the two coasts. Once paired according to these criteria, a coin was flipped for each pair to determine its allocation to the intervention or comparison arm. Kuujjuaq and Aupaluk were excluded from the randomisation: Kuujjuaq, because of its role as portal into the region (it was designated to receive mass screening without being entered into the randomisation); Aupaluk, because the nurse jumped the gun and when he heard about the possibility of screening decided on his own that his village should benefit from the screening.

Over the period March to May 1997, communities in the intervention arm received an intensive health education programme, and all sexually active adults were encouraged to give a first catch urine specimen for chlamydia screening. Young people were especially targeted in the health education programme, which used radio, newsletters, churches, local health promotion groups, and the village health care provider to convey the following messages:

- Take care of yourself and your partner; this means respecting your partner, making decisions together about physical intimacy.

- Use condoms every time you have intercourse, unless you know for sure that neither you nor your partner could have an STI.

- If there is a chance you could have an STI, go and get tested and treated.

Members of intervention communities were encouraged to bring a first catch urine specimen to their community health centre, where samples were stored at $-20^{\circ} \mathrm{C}$. Those in the age group 15 to 39 years were especially encouraged to participate in the screening programme. Specimens were transported by air to laboratories in Kuujjuaq and Montreal for testing using the Roche Amplicor Chlamydia PCR assay according to the manufacturer's instructions (Roche Diagnostic System, New Jersey, USA). An internal control was included with each test to monitor false negative results caused by inhibition. In order to measure the impact of the mass screening programme, specimens from routine prenatal screening and from women undergoing PAP smears were tested for $C$ trachomatis by both EIA and PCR in all communities. The impact was also measured by comparing reported rates of infection in intervention and comparison communities one year after mass screening.

Communities in the comparison arm continued with existing STI control services except that PCR testing was substituted for EIA. All individuals who tested positive for $C$ trachomatis were treated with $1 \mathrm{~g}$ azithromycin. 
Table 1 Participation rates by sex, and reported rates by village before and after screening

\begin{tabular}{|c|c|c|c|c|}
\hline \multirow[b]{2}{*}{ Village* } & \multicolumn{2}{|c|}{ Participation rates in 15-39 age group } & \multicolumn{2}{|c|}{ Reported chlamydia rates/1000 population } \\
\hline & Male (\%) & Female (\%) & $7 / 3 / 96-6 / 3 / 97$ & $7 / 7 / 97-6 / 7 / 98$ \\
\hline \multicolumn{5}{|c|}{ Intervention villages } \\
\hline 9 & $13 / 240(5)$ & $28 / 265(11)$ & 43 & 31 \\
\hline 12 & $7 / 85(8)$ & $28 / 65(43)$ & 49 & 27 \\
\hline 13 & $26 / 45(58)$ & $28 / 45(62)$ & 50 & 10 \\
\hline 11 & $25 / 95(26)$ & $67 / 110(61)$ & 33 & 18 \\
\hline 8 & $5 / 60(8)$ & $5 / 60(8)$ & 18 & 6 \\
\hline 10 & $52 / 220(24)$ & $69 / 175(39)$ & 33 & 35 \\
\hline Total & $183 / 1130$ (16) & $315 / 1075(29)$ & 37 & 24 \\
\hline \multicolumn{5}{|c|}{ Comparison villages } \\
\hline 1 & & & 31 & 18 \\
\hline 2 & & & 15 & 52 \\
\hline 3 & & & 15 & 14 \\
\hline 4 & & & 23 & 44 \\
\hline 5 & & & 31 & 27 \\
\hline 7 & & & 27 & 16 \\
\hline Total & & & 28 & 26 \\
\hline
\end{tabular}

Reported rates of infection before and after mass screening were compared using the Mantel-Haenszel $\chi^{2}$ test.

\section{RESULTS \\ Mass screening}

In all, 721 individuals were screened in six intervention communities with a total population of 5250, 2320 of whom were between 15 and 50 years of age. Coverage in the primary target population (aged 15 to 39 years) ranged from $8 \%$ to $60 \%$ in different communities. Over 700 urine specimens were tested for $C$ trachomatis by PCR. Eight per cent of male subjects tested positive, compared with $7 \%$ of female subjects. Table 1 shows the participation rates in the mass screening campaign by village and sex in the 15 to 39 year old age group, and the corresponding reported chlamydia rates per 1000 in the year before screening (EIA) and one year after it (PCR).

\section{Impact}

The reported rates of genital chlamydial infection in intervention communities decreased from 37.1/1000 population one year before the mass screening campaign to $24.2 / 1000$ one year after the screening period (odds ratio, 0.65; 95\% confidence interval 0.52 to $0.81 ; \mathrm{p}=0.0003$ ). The corresponding rates in comparison communities were $28.1 / 1000$ population before mass screening and 26.1 after the screening. In village 13, where the participation rates in the 15 to 19 and 20 to 24 age groups were $100 \%$ and $80 \%$, respectively, with a $1: 1$ male:female ratio, the reported chlamydia rates declined from $50 / 1000$ in the year preceding the mass screening campaign to $9.8 / 1000$ in the year after. In village 11 , although the participation rates were high, the male:female ratio was $1: 3$ and the corresponding decline in reported chlamydia rates was not as dramatic.

In the subset of women presenting for prenatal and PAP screening, the prevalence of chlamydial infection, determined by EIA, decreased from $12.3 \%$ before mass screening to $4.6 \%$ one year after screening $(\mathrm{p}=0.0002)$. The corresponding rates in comparison communities were $9.3 \%$ and $8.2 \%(p=0.63)$. Table 2 shows the chlamydia rates by age group in this population. There was a significant reduction in women aged 16 to 20 years $(p=0.02)$ and in those aged 21 to $25(p=0.003)$.

The ratio of women to men tested in the intervention communities fell from a mean of 4.3 in the four years preceding the mass screening campaign to 2.4 during the campaign and 2.6 in the year after the mass screening.
Table 2 Reported chlamydia rates by enzyme immunoassay in women presenting for prenatal care or PAP smear

\begin{tabular}{|c|c|c|c|c|c|}
\hline \multirow{2}{*}{$\begin{array}{l}\text { Age group } \\
\text { (years) }\end{array}$} & \multicolumn{2}{|c|}{ Prescreening } & \multicolumn{2}{|c|}{ Postscreening } & \multirow{2}{*}{$\begin{array}{l}p \text { Value }\left(\chi^{2}\right. \\
\text { test) }\end{array}$} \\
\hline & $\mathrm{n}$ & Rate & $\mathrm{n}$ & Rate & \\
\hline$<16$ & 42 & $11.9 \%$ & 24 & $8.3 \%$ & 0.65 \\
\hline $16-20$ & 381 & $14.7 \%$ & 188 & $7.3 \%$ & 0.02 \\
\hline $21-25$ & 428 & $19.6 \%$ & 200 & $5.5 \%$ & 0.003 \\
\hline $26-30$ & 372 & $5.7 \%$ & 157 & $6.4 \%$ & 0.75 \\
\hline Total & 1223 & $12.3 \%$ & 569 & $4.6 \%$ & 0.0002 \\
\hline
\end{tabular}

\section{DISCUSSION}

In terms of the phase specific concept of STI control, ${ }^{1}$ the epidemics of gonorrhoea and chlamydial infection in these communities are in phase II, being hyperendemic and generalised, although there is evidence that the incidence of gonorrhoea has started to decline following the introduction of a programme of health education, condom promotion, and screening in the early 1990s. The prevalence and incidence of chlamydial infection have remained intractably high, reflecting the fact that this infection is more often asymptomatic. In the absence of readily identifiable spread networks, screening programmes have been aimed at the general sexually active population, but in the past these have only targeted sexually active women. We have shown that a programme which also targets sexually active men, and tests a high proportion of the population over a short period of time using a highly sensitive assay, can have a major impact on the incidence and prevalence of chlamydial infection, even when coverage is suboptimal. If coverage could be substantially increased, which should not be impossible in these small communities, the impact would be greater and more sustained.

The high rates of suicide, substance abuse, and violence reflect the fact that these communities are under stress as a result of a breakdown in their traditional way of life, and the impact of western economic and social pressures. Similarly high STI rates have been reported in other aboriginal communities under similar conditions, including both Inuit and nonInuit populations. ${ }^{67}$ The unique feature of these Inuit communities is their small size and isolation; in theory, it should be feasible to eliminate treatable STIs from such communities if it were possible to identify a high proportion of cases and provide them with effective treatment on a given day. 
As resources for screening are available in Canada, and after discussion with community leaders, we opted for mass screening, with treatment of infected cases, as the most appropriate strategy for the control of chlamydial infection in this setting. When resources for laboratory testing are not available, alternative strategies have been tried for the control of STI epidemics in the hyperendemic phase, including selective mass treatment of high risk groups, and mass treatment of entire communities.

Selective mass treatment for gonorrhoea (benzyl penicillin + probenecid) was given to Inuit communities in Greenland in the 1960s. ${ }^{6}$ In southern Greenland, the anti-gonorrhoea campaign was carried out by a trained team, who supplemented mass treatment with a clinical examination and laboratory diagnosis using a mobile laboratory, and traced contacts. In the rest of the country, treatment was given without examination or laboratory diagnosis to all unmarried individuals aged 15 to 30 years, and all those who had been diagnosed with gonorrhoea in the previous six months. The coverage achieved was not stated.

The initial prevalence of gonorrhoea among those examined at baseline in southern Greenland (in May 1965) was 23\% in the towns and $17 \%$ in the smaller settlements. At subsequent examinations between November 1966 and May 1968, the prevalence in the towns was between $10 \%$ and $14 \%$ and in the settlements between $2 \%$ and $5 \%$. The authors concluded that this campaign was more successful in small remote communities. The campaign had little impact on the number of reported cases of gonorrhoea in either southern Greenland or the rest of the country.

Selective mass treatment is more effective when it can be targeted to readily identifiable core groups who constitute the main reservoir of infection, corresponding to phase 3 in the dynamic topology model of STI transmission. Condom promotion campaigns among sex workers in Nairobi, Kenya, for example, has reduced the impact of HIV infection and other STIs, and monthly presumptive treatment of sex workers is currently being evaluated in that population. ${ }^{8}$

Mass treatment of the entire adult population, delivered every 10 months, had little impact on STI rates in Uganda. ${ }^{9}$ After two rounds of mass treatment, with a coverage of $70-80 \%$, the prevalence of $T$ vaginalis infection was $9.3 \%$ in the intervention communities $v 14.4 \%$ in the comparison communities $(\mathrm{p}<0.05)$, and the prevalence of serological syphilis was $5.6 \% \vee 6.8 \%(\mathrm{p}<0.05)$; but there was no significant difference in the prevalence of any other STI between the two arms. The low impact of mass treatment in Greenland and Uganda can presumably be attributed to the mobility of the target populations, allowing STIs to be reintroduced from outside the treated area.

The best way to control STIs in the hyperendemic phase remains to be determined. Where spread networks can be identified, strategies which target them are likely to be most cost-effective. In communities such as we describe, services should be provided for the entire population but special efforts should be made to reach young people, and particularly young men. Women are often well aware of the dangers of promiscuity, but powerless to persuade their male partners to use condoms. ${ }^{3}$ Health education and condom promotion must be given high priority in the medium to long term. In the short term, screening programmes using sensitive non-invasive tests offer an opportunity to reduce the prevalence of treatable STIs, but the optimal strategy for their deployment remains to be determined. Should mass screening programmes, in which a high coverage is achieved over a short period of time, be organised on a regular basis? And if so, how often? Or would it be more cost-effective to encourage people to be screened on a continuing regular basis? The problem here would be to reach men, who usually have less contact with health services than women. Innovative programmes could be developed in which screening is offered outside health facilities. Mathematical modelling may have a role to play in predicting the most effective strategies for the control of hyperendemic STIs, but there is also a great need for intervention trials which measure the impact of different interventions in high prevalence populations.

Authors' affiliations

S Hodgins, S Dery, J-F Proulx, Nunavik Board of Health, Kuujiuaq,

Quebec, Canada

R W Peeling, National Laboratory for Sexually Transmitted Diseases, Health Canada, Winnipeg, Canada

F Bernier, Hema Quebec, Montreal, Canada

J Joly, Laboratoire de la Santé Publique de Québec, Montreal, Canada

M Alary, A LaBrecque, Laval University, Québec, Canada

D Mabey, London School of Hygiene and Tropical Medicine, London WC1, UK

\section{REFERENCES}

1 Wasserheit JN, Aral SO. The dynamic topology of sexually transmitted disease epidemics: implications for prevention strategies. J Infect Dis 1996; 174(suppl 2):S201-3

2 Jetté $M$, ed. A health profile of the Invit: report of the Santé Québec health survey among the Inuit of Nunavik, 1992, vol III. Montreal: Ministère de la Santé et des Services sociaux du Québec, 1995.

3 Labrecque A. Les perceptions des maladies transmissibles sexuellement chez des femmes inuites, selon le modele de continuite. Memoire du grade de Maitre en sciences infirmieres. Montreal: Université de Montréal, 1996. [MSc thesis.]

4 Grosskurth H, Mosha F, Todd J, et al. Impact of improved treatment of sexually transmitted diseases on HIV infection in rural Tanzania: randomised controlled trial. Lancet 1995;346:530-6.

5 Cohen MJ. Sexually transmitted diseases facilitate HIV transmission: no longer a hypothesis. Lancet 1998;351 (Suppl III):57.

6 Olsen GA. Epidemiological measures against gonorrhoea. Experience in Greenland. Br J Vener Dis 1973;49:130-3

7 Miller PJ, Torzillo PJ, Hateley W. Impact of improved diagnosis and treatment on prevalence of gonorrhoea and chlamydial infection in remote Aboriginal communities on Anangu and Pitjantjatjara Lands. Med J Aust 1999:170:429-32

8 Fonck K, Kaul R, Kimani J, et al. A randomized, placebo-controlled trial of monthly azithromycin prophylaxis to prevent sexually transmitted infections and HIV-1 in Kenyan sex workers: study design and baseline findings. Int J STD AIDS 2000;11:804-11.

9 Wawer MJ, Sewankambo NK, Serwadda D, et al. Control of STDs for AIDS prevention in Uganda: a randomised community trial. Lancet 1999;353:525-35. 